\title{
A candidate gene study of the type I interferon pathway implicates IKBKE and IL8 as risk loci for SLE
}

\author{
Johanna K Sandling ${ }^{1}$, Sophie Garnier ${ }^{1,12}$, Snaevar Sigurdsson ${ }^{1,13}$, Chuan Wang ${ }^{1}$, Gunnel Nordmark ${ }^{2}$, \\ Iva Gunnarsson ${ }^{3}$, Elisabet Svenungsson ${ }^{3}$, Leonid Padyukov ${ }^{3}$, Gunnar Sturfelt ${ }^{4}$, Andreas Jönsen ${ }^{4}$, Anders \\ A Bengtsson ${ }^{4}$, Lennart Truedsson ${ }^{5}$, Catharina Eriksson ${ }^{6}$, Solbritt Rantapää-Dahlqvist ${ }^{7}$, Anders Mälarstig ${ }^{8}$, \\ Rona J Strawbridge ${ }^{8}$, Anders Hamsten ${ }^{8}$, Lindsey A Criswell ${ }^{9}$, Robert R Graham ${ }^{10}$, Timothy W Behrens ${ }^{10}$, \\ Maija-Leena Eloranta ${ }^{2}$, Gunnar Alm ${ }^{11}$, Lars Rönnblom ${ }^{2}$ and Ann-Christine Syvänen ${ }^{\star, 1}$
}

Systemic Lupus Erythematosus (SLE) is a systemic autoimmune disease in which the type I interferon pathway has a crucial role. We have previously shown that three genes in this pathway, IRF5, TYK2 and STAT4, are strongly associated with risk for SLE. Here, we investigated 78 genes involved in the type I interferon pathway to identify additional SLE susceptibility loci. First, we genotyped 896 single-nucleotide polymorphisms in these 78 genes and 14 other candidate genes in 482 Swedish SLE patients and 536 controls. Genes with $P<0.01$ in the initial screen were then followed up in 344 additional Swedish patients and 1299 controls. SNPs in the IKBKE, TANK, STAT1, IL8 and TRAF6 genes gave nominal signals of association with SLE in this extended Swedish cohort. To replicate these findings we extracted data from a genomewide association study on SLE performed in a US cohort. Combined analysis of the Swedish and US data, comprising a total of 2136 cases and 9694 controls, implicates IKBKE and IL8 as SLE susceptibility loci $\left(P_{\text {meta }}=0.00010\right.$ and $P_{\text {meta }}=0.00040$, respectively). STAT1 was also associated with SLE in this cohort $\left(P_{\text {meta }}=3.3 \times 10^{-5}\right)$, but this association signal appears to be dependent of that previously reported for the neighbouring STAT4 gene. Our study suggests additional genes from the type I interferon system in SLE, and highlights genes in this pathway for further functional analysis.

European Journal of Human Genetics (2011) 19, 479-484; doi:10.1038/ejhg.2010.197; published online 22 December 2010

Keywords: systemic lupus erythematosus; type I interferon system; candidate gene study; single nucleotide polymorphism; IKBKE; IL8

\section{INTRODUCTION}

Systemic Lupus Erythematosus (SLE, OMIM 152700) is an inflammatory autoimmune disease that primarily affects women during their childbearing years. Production of autoantibodies, tissue deposits of immune complexes and inflammation in kidneys, skin, joints and central nervous system are hallmarks of SLE. Despite a strong heritability of the disease, linkage studies have failed to identify genes outside the major histocompatibility complex (MHC) region as risk factors for SLE. Association studies have since proven a more fruitful approach. The confirmed findings from genomewide association studies (GWAS) include genes that were originally discovered in candidate gene studies, such as the interferon regulatory factor 5 $(\text { IRF5) })^{1}$ and the signal transducer and activator of transcription 4 $(S T A T 4)^{2}$ genes from the type I IFN system. IFNs are cytokines with antiviral activity that are produced in response to viral infections, of which the type I IFNs bind the IFN- $\alpha$ receptor (IFNAR). Today there are more than 20 confirmed SLE susceptibility loci, ${ }^{3,4}$ of which several are in the type I IFN system.

The type I IFN system is activated in SLE patients, ${ }^{5,6}$ and an important role of the type I IFN system in the disease process was confirmed by studies showing an increased expression of type I IFN-inducible genes in SLE patients (an 'IFN-signature'). ${ }^{7}$ A direct causative role of the type I IFN system in the etiopathogenesis of SLE was suggested by the observation that individuals treated with IFN- $\alpha$ can develop an SLE syndrome indistinguishable from the naturally occurring disease. ${ }^{8}$ Moreover, a phase I clinical trial with a monoclonal antibody against IFN- $\alpha$, reports reduction of disease activity as well as neutralization of the IFN signature in SLE patients. ${ }^{9,10}$ Encouraged by the compelling evidence for the involvement of the type I IFN system in SLE, we performed an association study to identify additional genes from the type I IFN pathway that confer risk for SLE.

\footnotetext{
${ }^{1}$ Molecular Medicine, Department of Medical Sciences, Uppsala University, Uppsala, Sweden; ${ }^{2}$ Section of Rheumatology, Department of Medical Sciences, Uppsala University, Uppsala, Sweden; ${ }^{3}$ Rheumatology Unit, Department of Medicine, Karolinska Institutet/Karolinska University Hospital, Stockholm, Sweden; ${ }^{4}$ Section of Rheumatology, Department of Clinical Sciences, Lund University, Lund, Sweden; ${ }^{5}$ Department of Laboratory Medicine, section of M.I.G., Lund University, Lund, Sweden; ${ }^{6}$ Department of Clinical Immunology, Umeå University Hospital, Umeå, Sweden; ${ }^{7}$ Department of Rheumatology, Umeå University Hospital, Umeå, Sweden; ${ }^{8}$ Atherosclerosis Research Unit, Department of Medicine, Solna Karolinska Institutet Stockholm, Stockholm, Sweden; ${ }^{9}$ Rosalind Russell Medical Research Center for Arthritis, Department of Medicine, University of California, San Francisco, CA, USA; ${ }^{10}$ Genentech Inc., South San Francisco, CA, USA; ${ }^{11}$ Department of Biomedical Sciences and Veterinary Public Health, Swedish University of Agricultural Sciences, Uppsala, Sweden

*Correspondence: Professor A-C Syvänen, Department of Medical Sciences, Uppsala University, Molecular Medicine, Entrance 70, Research Department 2, 3rd floor, Uppsala University Hospital, 75185 Uppsala, Sweden. Tel: +46 18 6112959; Fax: +46 185536 01; E-mail: Ann-Christine.Syvanen@medsci.uu.se

${ }^{12}$ Current address: INSERM UMR S937, Génétique Epidémiologique et Moléculaire des Pathologies Cardiovasculaires, Université Paris VI, Paris, France.

${ }^{13}$ Current address: Broad Institute of Harvard and MIT, Boston, MA, USA.
}

Received 5 May 2010; revised 9 August 2010; accepted 8 October 2010; published online 22 December 2010 


\section{MATERIALS AND METHODS}

\section{Subjects and genotyping}

Our study included 826 Swedish and 1310 US SLE patients, fulfilling at least four of the classification criteria for SLE as defined by the American College of Rheumatology (ACR), ${ }^{11}$ and 9694 healthy control individuals from the same geographic areas as the patients (Supplementary Table S1). DNA was extracted from blood samples of the patients and controls using standard procedures. The study was approved by the regional ethical boards and all subjects gave their informed consent to participate. The study was performed in three stages:

Discovery phase. First, a panel of SNPs in 82 genes with key functions related to the type I IFN signalling system and 14 additional genes, with suggested association with SLE, were selected for genotyping in 490 Swedish SLE patients and 543 controls. Patients were from the rheumatology clinics at the Lund, Karolinska (Solna) and Uppsala University Hospitals in Sweden. SNPs were selected on the basis of their average spacing of around $1 \mathrm{~kb}$ and LD information from the HapMap project $\left(r^{2}<0.8\right.$ HapMap CEU release 16c), excluding SNPs with an Illumina quality score $<0.6$. Genotyping of 1258 SNPs in the 96 genes was performed using the Golden Gate Assay (Illumina Inc., San Diego, CA, USA). Samples and SNPs with $>10 \%$ missing data, SNPs with Hardy-Weinberg equilibrium test $P$-values $<0.001$ and SNPs with MAF $<0.01$ were excluded from further analysis. Four parent-offspring trios were included in the genotyping for inheritance checks, and no Mendelian inheritance errors were observed. After exclusion of genetic outliers, duplicate or related samples 482 cases, 536 controls and 896 SNPs in 92 genes were available for analysis.

Confirmatory set. In the second phase, SNPs in the eight most promising genes were followed up in 393 patients and 1645 controls from Sweden. Patients were from the rheumatology clinics at the Umeå, Uppsala and Karolinska (Solna) University Hospitals. For the patients and 972 of the controls, genotyping of 25 SNPs was performed using the 12-plex and 48-plex SNPStream systems ${ }^{12}$ (Beckman Coulter Inc., Brea, CA, USA). Primer sequences are provided in Supplementary Table S2. The same quality control filters as for the discovery cohort were applied, and samples overlapping with the discovery phase were excluded. Additional Swedish population-based controls from the Stockholm area $(n=673)$, previously genotyped using the Infinium II assay on human $1 \mathrm{M} v 1$ bead arrays ${ }^{13}$ (Illumina Inc.), were also included after applying the following quality filters: MAF $>1 \%$, HWE $P>1 \times 10^{-6}$, SNP and sample call rates $>95 \%$. After exclusion of genetic outliers, duplicate or related samples, a total of 344 cases, 1299 controls and 21 SNPs were available for analysis.

GWAS. We sought replication of our results by using data from a GWAS on SLE in US Caucasians. ${ }^{14}$ In brief, 1435 North-American SLE cases of European descent and 3583 controls had been genotyped on HumanHap550 bead arrays (Illumina Inc.). An additional 4564 controls were also included as previously described. ${ }^{4}$ After strict quality control ${ }^{14} 1310$ cases and 7859 controls remained.

\section{Additional quality control and imputation}

For the 673 Swedish controls genotyped on the $1 \mathrm{M}$ bead arrays, genotypes for 13 confirmatory phase SNPs that were not directly genotyped were imputed using the software IMPUTE and phasing data from the HapMap project. ${ }^{15}$ In the US GWAS dataset genotypes were imputed for 15 SNPs using the IMPUTE software. SNPs had imputation confidence scores $\geq 0.90$ with one exception (rs4694178, confidence 0.85 in the US data). For all the Swedish SLE patients, and for more than half of the Swedish controls genotyped using the GoldenGate or SNPstream methods, data from 6060 uncorrelated ancestry informative markers (AIMs) became available during the course of our study. ${ }^{4}$ Using this data, genetic outliers were identified with principal component analysis by the EIGENSTRAT software ${ }^{16}$ and excluded from the study (the ten first principal components were inferred and a cut-off of $\sigma>6$ was used to identify outliers). These samples were also checked for cryptic relatedness by investigation of identity-by-state (IBS) status in PLINK ${ }^{17}$ (http://pngu.mgh. harvard.edu/purcell/plink/) using a set of $12 \mathrm{k}$ previously genotyped SNPs. For the Swedish controls, genotyped on the $1 \mathrm{M}$ arrays, all markers were used to identify cryptically related samples and 6035 successfully genotyped AIMs were used to identify genetic outliers.

\section{Association analysis and power calculation}

The association analysis of the directly genotyped SNPs was performed by comparing allele frequencies in cases and controls with Fisher's exact or Chi2 tests using PLINK. A null distribution for the quantile-quantile (Q-Q) plot was generated with PLINK and plotted using R (http://www.r-project.org/). Analyses including imputed genotypes were performed using SNPTEST, ${ }^{15}$ which takes imputation uncertainty into account. SNPs, which were not captured by imputation were only analysed in the directly genotyped samples. Conditional logistic regression analysis assuming an additive model was performed using PLINK to test for independence of association signals observed in neighbouring genes, or between associated SNPs within the same gene. Tests for pairwise SNP interactions were performed using the epistasis command in PLINK. The combined analysis of the Swedish and US casecontrol data was performed using the software Metal (http://www.sph.umich. edu/csg/abecasis/Metal/index.html). Pooled odds ratios were calculated using the Mantel-Haenszel method under a fixed effects model, and tests for heterogeneity of odds ratios between studies were calculated using the MedCalc software (http://www.medcalc.be/). Power calculations were performed using the software QUANTO (http://hydra.usc.edu/GxE/) assuming a log-additive model, and a prevalence for SLE in Sweden of $0.05 \%$.

\section{RESULTS}

We selected a panel of SNPs in 78 genes with key functions related to the type I IFN signalling system to study their association with SLE in a Swedish case-control cohort. The selected genes encode Toll-like receptors (TLRs) and intracellular sensor molecules for nucleic acids (ie RIG-I-like receptors: RLR) and members of their signalling pathways, including several transcription factors that are active in the IFN producing pDCs and membrane proteins of the pDCs. Genes encoding the members of the type I IFN family and other genes regulated by the TLRs that are involved in the response to the type I and type III IFNs, and genes for which the expression is directly regulated by type I IFNs, were also included in the panel. Although the type I and type III IFNs bind two different receptors, the IFNAR and IFN- $\lambda$ receptor, they share downstream signalling and IFN- $\lambda$-induced genes are also induced by type I IFNs. A total of 14 additional genes that are not directly involved in the type I IFN system, but have been suggested to be associated with SLE were also included (Supplementary Table S3). This analysis identified 21 SNPs in seven genes that yielded unadjusted $P$-values $<0.01$ : IKBKE, TANK, STAT1, IL8, NRP1, TRAF6 and PIAS4 (Figure 1 and Supplementary Table S3). At this significance level only nine associated SNPs would be expected to yield association signals by chance, which indicates the presence of true association signals in our data (Supplementary Figure S1). The power for the discovery phase, which included 482 SLE cases and 536 controls, was $65 \%$ to detect an OR of 1.5 at 0.01 significance for a $10 \%$ frequency allele. However, our power to detect genes with an OR of 1.2 was considerably lower (10\%), and thus we cannot exclude that genes, which remained undetected in our study may contribute to the risk for SLE.

To increase power, we designed a panel of SNPs in the IKBKE, TANK, STAT1, IL8, NRP1, TRAF6 and PIAS4 genes for genotyping in an independent collection of Swedish SLE patients and controls. The STAT5B gene was also included in the follow-up study, as initially one SNP in the gene showed a $P$-value $<0.01$. This was, however, before an additional quality control step, by which related samples and population outliers were excluded. For genes, where multiple SNPs showed $P$-values $<0.05$ in the discovery phase, candidate SNPs for follow-up were tested for independence of their association signal in relation to the most strongly associated SNP in each gene (Supplementary Table S4). A partly redundant set of 21 SNPs that accounted for the association signals from the discovery phase was then analysed in the second Swedish case-control cohort. Also in this 


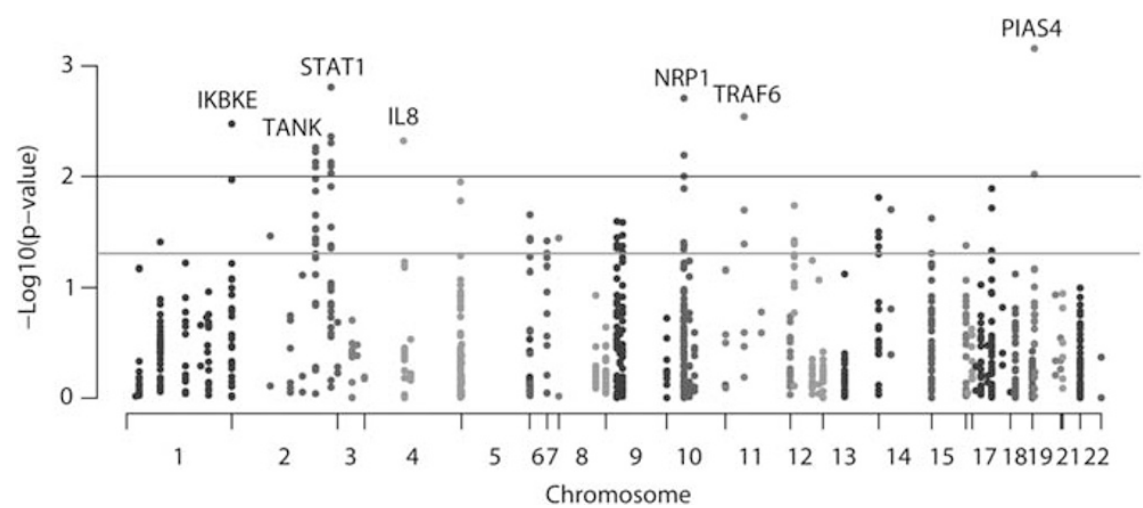

Figure 1 Association with SLE for the 92 genes tested in the discovery phase (482 Swedish cases and 536 controls). The negative logarithm of the $P$-value from a $\chi^{2}$ - test is plotted against chromosomal location. The grey line represents $P=0.05$, and the black line $P=0.01$.

Table 1 Combined analysis of association with SLE in the Swedish discovery and confirmation cohorts ${ }^{\mathrm{a}}$

\begin{tabular}{|c|c|c|c|c|c|c|c|c|c|}
\hline Gene & $\mathrm{CHR}$ & $S N P^{\mathrm{b}}$ & Basepair & Minor/major alleles & MAF cases & MAF controls & $\mathrm{P}^{\mathrm{c}}$ & Risk allele & OR $(95 \% \mathrm{Cl})$ \\
\hline$I K B K E$ & 1 & rs1539243 & 204714410 & $\mathrm{~T} / \mathrm{C}$ & 0.16 & 0.18 & 0.031 & C & $1.19(1.02-1.39)$ \\
\hline IKBKE & 1 & rs17433930 & 204719362 & $\mathrm{G} / \mathrm{A}$ & 0.07 & 0.09 & 0.013 & A & $1.33(1.06-1.67)$ \\
\hline TANK & 2 & rs1267075 & 161768063 & $\mathrm{G} / \mathrm{A}$ & 0.19 & 0.18 & 0.75 & G & $1.02(0.88-1.19)$ \\
\hline STAT1 & 2 & rs2030171 & 191577408 & $\mathrm{~A} / \mathrm{G}$ & 0.35 & 0.29 & $1.2 \mathrm{E}-05$ & A & $1.31(1.16-1.49)$ \\
\hline STAT1 & 2 & rs16833172 & 191584314 & $\mathrm{~A} / \mathrm{G}$ & 0.04 & 0.03 & 0.015 & A & $1.51(1.09-2.09)$ \\
\hline$N R P 1$ & 10 & rs1331314 & 33564687 & $\mathrm{G} / \mathrm{C}$ & 0.11 & 0.10 & 0.32 & G & $1.11(0.92-1.34)$ \\
\hline NRP1 & 10 & rs2073320 & 33593263 & $A / G$ & 0.38 & 0.37 & 0.42 & A & $1.05(0.93-1.18)$ \\
\hline TRAF6 & 11 & rs5030482 & 36466602 & $\mathrm{C} / \mathrm{T}$ & 0.12 & 0.14 & 0.0094 & $\mathrm{~T}$ & 1.25 (1.05-1.49) \\
\hline TRAF6 & 11 & rs5030472 & 36470362 & $\mathrm{~T} / \mathrm{C}$ & 0.09 & 0.12 & 0.013 & C & $1.28(1.05-1.55)$ \\
\hline STAT5B & 17 & rs6503691 & 37647616 & $\mathrm{~T} / \mathrm{C}$ & 0.09 & 0.10 & 0.44 & C & $1.08(0.88-1.32)$ \\
\hline PIAS4 & 19 & rs2289863 & 3979783 & $\mathrm{C} / \mathrm{T}$ & 0.27 & 0.29 & 0.19 & $\mathrm{~T}$ & $1.09(0.96-1.25)$ \\
\hline
\end{tabular}

Abbreviations: $\mathrm{Cl}$, confidence Interval; MAF, minor Allele Frequency; OR, odds ratio; SNP, single-nucleotide polymorphism.

a 826 Swedish cases and 1835 controls genotyped in the discovery and replication phases including controls genotyped on the $1 \mathrm{M}$ chip.

bGenotypes for rs17433930, rs3754974, rs2030171, rs16833172, rs4694178, rs734187 and rs1331314 were imputed in the Swedish controls genotyped on the 1M chip.

c $P$-values calculated using SNPtest.

$P$-values $<0.05$ are indicated in bold.

confirmatory cohort genetic outliers were excluded. By combining the data from the Swedish discovery and confirmatory cohorts, totalling 826 cases and 1835 controls, we observed strong association signals with $P<5 \times 10^{-4}$ for the SNPs rs2030171 in STAT1 and rs4694178 in IL8 (Table 1 and Supplementary Table S5). Multiple linked SNPs in the TRAF family member-associated NF $\kappa$ B activator (TANK) and TNF receptor-associated factor 6 (TRAF6) genes also gave suggestive signals of association with SLE in the combined Swedish cohort. In addition, two weakly correlated SNPs in the IKBKE gene (rs1539243 and rs17433930, LD $r^{2}=0.34$ ) displayed $P$-values $<0.05$. Conditional regression analysis did not provide evidence for more than one allele contributing to risk for SLE in genes with multiple associated SNPs (Supplementary Table S5), nor was there any evidence for epistatic interactions between SNPs at different loci.

We sought independent replication of our results by using data from a GWAS on SLE in European Americans. ${ }^{14}$ The SNPs, rs1539243 and rs17433930, in the IKBKE gene showed signals of association with SLE also in the US data $(P=0.0028$ and $P=0.0021$, respectively) (Table 2 and Supplementary Table S6). The SNP rs1539243 had been directly genotyped in the US cohort, and for rs17433930 imputed genotypes were analysed. Combined analysis of the data from the
Swedish and US cohorts revealed convincing association signals with SLE for these SNPs $\left(P_{\text {meta }}=0.00026, \mathrm{OR}=1.19\right.$, and $P_{\text {meta }}=0.00010$, $\mathrm{OR}=1.33$, for rs1539243 and rs17433930, respectively), a result which was significant also after Bonferroni correction $\left(P_{\text {meta_corr }}<0.01\right)$. The two IKBKE SNPs rs17433930 and rs1539243, which we found to be associated with SLE are located in the tenth intron and fourth exon of the gene, respectively, where rs1539243 is a synonymous SNP in amino-acid residue 67 (Ile) of the IKK $\varepsilon$ kinase.

Although the SNP rs4694178, located $3.3 \mathrm{~kb}$ downstream of the IL8 gene, had only a trend-wise significant $P$-value in the US cohort compared with the more convincing association with SLE in the Swedish cohort $\left(P_{\mathrm{US}}=0.064, P_{\mathrm{SWE}}=5 \times 10^{-5}\right)$, its association signal remained significant in the combined analysis after Bonferroni correction $\left(P_{\text {meta }}=0.00040, \mathrm{OR}=1.17, P_{\text {meta_corr }}<0.01\right) \quad$ (Table 2). This SNP was imputed in the US data (confidence $=0.85$ ), however, the directly genotyped SNP rs9999446, which is strongly correlated with the SNP rs4694178 $\left(r^{2}=0.86\right)$ yielded a similar result $(P=0.085)$ for association with SLE. For the SNP rs10199181 in the STAT1 gene, we observed combined $P$-values $<1 \times 10^{-3}$. The STAT1 gene is located close to the STAT4 gene in a region of high LD on chromosome 2q32.2. STAT4 contains two linked SNPs, rs10181656 and rs7582694, which are strongly associated with SLE, ${ }^{18}$ and 
Table 2 Meta-analysis of association with SLE for the Swedish and US cohorts

\begin{tabular}{|c|c|c|c|c|c|c|c|c|c|}
\hline \multirow[b]{2}{*}{ Gene } & \multirow[b]{2}{*}{ SNP } & \multicolumn{2}{|c|}{ Sweden ${ }^{\mathrm{a}}$} & \multicolumn{2}{|r|}{$U S^{b}$} & \multicolumn{4}{|c|}{ Meta-analysis $^{c}$} \\
\hline & & $P^{d}$ & OR $(95 \% \mathrm{Cl})$ & $P^{d}$ & OR $(95 \% C l)^{\mathrm{e}}$ & Risk allele & $P$ & Corrected $\mathrm{P}^{\mathrm{f}}$ & OR $(95 \% C l)^{\mathrm{e}}$ \\
\hline IKBKE & rs1539243 & 0.031 & $1.19(1.02-1.39)$ & 0.0028 & $1.20(1.07-1.35)$ & C & 0.00026 & 0.0054 & $1.19(1.09-1.31)$ \\
\hline TANK & rs3754974 & 0.0087 & $1.44(1.09-1.89)$ & 0.98 & $0.99(0.80-1.22)$ & NA & 0.22 & 1.0 & $1.13(0.96-1.34)$ \\
\hline TANK & rs1267075 & 0.75 & $1.02(0.88-1.19)$ & 0.87 & $0.99(0.89-1.11)$ & NA & 0.99 & 1.0 & $1.00(0.92-1.10)$ \\
\hline STAT1 & rs2030171 & $1.2 \mathrm{E}-05$ & $1.31(1.16-1.49)$ & 0.018 & $1.11(1.02-1.21)$ & $A$ & $3.3 \mathrm{E}-05$ & 0.00069 & $1.17(1.09-1.26)$ \\
\hline$N R P 1$ & rs734187 & 0.67 & $1.03(0.90-1.18)$ & 0.66 & $1.01(0.92-1.12)$ & A & 0.56 & 1.0 & $1.02(0.94-1.10)$ \\
\hline$N R P 1$ & rs1331314 & 0.32 & $1.11(0.92-1.34)$ & 0.11 & $1.11(0.97-1.26)$ & $G$ & 0.061 & 1.0 & $1.11(1.00-1.23)$ \\
\hline$N R P 1$ & rs2073320 & 0.42 & $1.05(0.93-1.18)$ & 0.61 & $0.98(0.90-1.06)$ & NA & 0.94 & 1.0 & $1.00(0.94-1.07)$ \\
\hline TRAF6 & rs5030482 & 0.0094 & $1.25(1.05-1.49)$ & 0.12 & $1.10(0.97-1.24)$ & $\mathrm{T}$ & 0.0097 & 0.20 & $1.14(1.03-1.26)$ \\
\hline TRAF6 & rs5030472 & 0.013 & $1.28(1.05-1.55)$ & 0.19 & $1.09(0.96-1.25)$ & $\mathrm{C}$ & 0.020 & 0.42 & $1.15(1.03-1.28)$ \\
\hline STAT5B & rs6503691 & 0.44 & $1.08(0.88-1.32)$ & 0.78 & $0.98(0.86-1.12)$ & NA & 0.91 & 1.0 & $1.01(0.90-1.13)$ \\
\hline
\end{tabular}

Abbreviations: $\mathrm{CI}$, confidence Interval; MAF, minor Allele Frequency; NA, not Available; OR, odds ratio; SNP, single-nucleotide polymorphism.

a826 Swedish cases and 1835 controls genotyped in the discovery and replication phases including controls genotyped on the $1 \mathrm{M}$ chip.

b1310 US SLE cases and 7859 controls genotyped in a GWAS. Genotypes for rs17433930, rs3754974, rs2030171, rs16833172, rs4694178, rs734187, rs1331314 and rs5030482 were

imputed in this dataset as described in Gateva et al.

cMeta-analysis including 2136 SLE cases and 9694 controls.

d $P$-values calculated using SNPtest.

eOdds ratios are calculated relative to the risk allele defined in the analysis of the combined Swedish cohort.

${ }^{\mathrm{f}} P$-value corrected for the 21 tested SNPs.

$P$-values $<0.05$ are indicated in bold.

conditional regression analysis of the data from the combined Swedish cohort indicates that the association signals from the STAT1 and STAT4 SNPs are not independent of each other (data not shown, remaining $P_{\text {conditional }}>0.3$ ).

\section{DISCUSSION}

Our association study of genes from the type I IFN pathway and additional candidate genes for SLE highlighted two genes, IKBKE and $I L 8$, as potential risk factors for SLE. In addition, the genes TRAF6 and TANK showed significant association with SLE in the Swedish cohorts. Furthermore, IRF5, TYK2, STAT4, IFIH1, IRAK1, IRF8 and the PHRF1/IRF7 region have been reported by us elsewhere to be associated with SLE. ${ }^{1,4,18}$ Thus, polymorphisms in multiple genes connected to the type I IFN signalling system are important for SLE disease susceptibility.

$I K B K E$, the inhibitor of nuclear factor kappa-B kinase subunit epsilon gene, encodes IKK $\varepsilon$, a kinase that together with the TANKbinding kinase (TBK1) has a role in the innate antiviral response. IKK $\varepsilon$ and TBK1 are activated when two intracellular RLR helicases, encoded by the IFIH1 and DDX58 genes, recognise viral RNA in virus-infected cells (Figure 2). These kinases are also activated upon stimulation of endosomal TLR3 by double stranded DNA, or cell membrane TLR4 by bacterial lipopolysaccharide (LPS). Together with TBK1, IKK $\varepsilon$ mediates phosphorylation of the transcription factors IRF3 and IRF7, which leads to their activation and subsequent transcription of type I IFN and other inflammatory cytokines, but also activation of NF $\kappa$ B has been reported. ${ }^{19,20}$ The activation of IRF3 and IRF7 can be inhibited by an ubiquitin-editing enzyme (A20), encoded by the TNFAIP3 gene. As also variation in TNFAIP3 and IFIH1 are associated with SLE, ${ }^{4,21,22}$ this further supports an important role for the RLR pathway in the disease process. There is also evidence that IKK $\varepsilon$ can phosphorylate STAT1, and thus contribute to the type I IFN signalling through the IFNAR. ${ }^{23}$

The IKBKE gene has recently been implicated in risk for rheumatoid arthritis (RA). ${ }^{24}$ The two polymorphisms with the most significant association with RA were tested in the discovery phase of our study (rs2151222 and rs3748022 with $P=0.084$ and $P=0.49$ for association to SLE, respectively), however, these variants appear to be independent from the IKBKE variants rs1539243 and rs17433930 that we identified as risk alleles for SLE $\left(r^{2}<0.1\right.$ discovery phase). The conjecture that IKBKE has a role in arthritis is supported by data from an animal model. IKKe knockout mice have been shown to be less sensitive to induction of arthritis and exhibit less joint destruction than control mice. ${ }^{25}$ In a published GWAS on women with $\mathrm{SLE}^{26}$ the IKBKE SNP rs1539243 was tested, but no association was observed (dbGaP, http://www.ncbi.nlm.nih.gov/gap). The power of that study was, however, only $23 \%$ at the $P=0.05$ level to detect the association we observe.

The chemokine IL- 8 has a wide range of pro-inflammatory effects, and its production can be triggered by immune complexes that also have the capacity to induce type I IFN production. ${ }^{27}$ Recent data also suggests that IL-8 production in virus-infected cells is IFN dependant. ${ }^{28}$ SLE patients with renal ${ }^{29}$ or CNS involvement ${ }^{30}$ have elevated IL-8 levels in their serum and cerebrospinal fluid, respectively, and serum IL-8 levels and disease activity correlate in SLE patients. ${ }^{31}$ As SLE disease flares are associated with increased IFN- $\alpha$ production, ${ }^{6}$ these observations provide a link between the SLE disease process, IL- 8 and the type I IFN system.

Although we observed a significant association of IL8 with SLE in this study, there are previous conflicting reports on the association between IL8 and SLE, ${ }^{26,32-34}$ only two of which, a Spanish case-control study and a GWAS in women with SLE, having similar power to our discovery phase. The power of these studies was around $50 \%$ at the 0.05 significance level to detect the effect for IL8 that we observe. The LD between the SNP rs2227306 tested in the Spanish study and the SNP rs4694178 in our study is very high $\left(r^{2}=0.97\right.$ in the HapMap CEU population), but they do not observe any association with SLE for this SNP. ${ }^{34}$ In our study, the association signal is mainly contributed by the Swedish cohort, which has a higher frequency of the risk allele than both the US and Spanish cohorts (control frequency of rs4694178 C: Sweden $=0.46, \mathrm{USA}=0.41$ and the linked allele rs2227306 T: Spain=0.41). In the women $\mathrm{GWAS}^{26}$ a SNP 


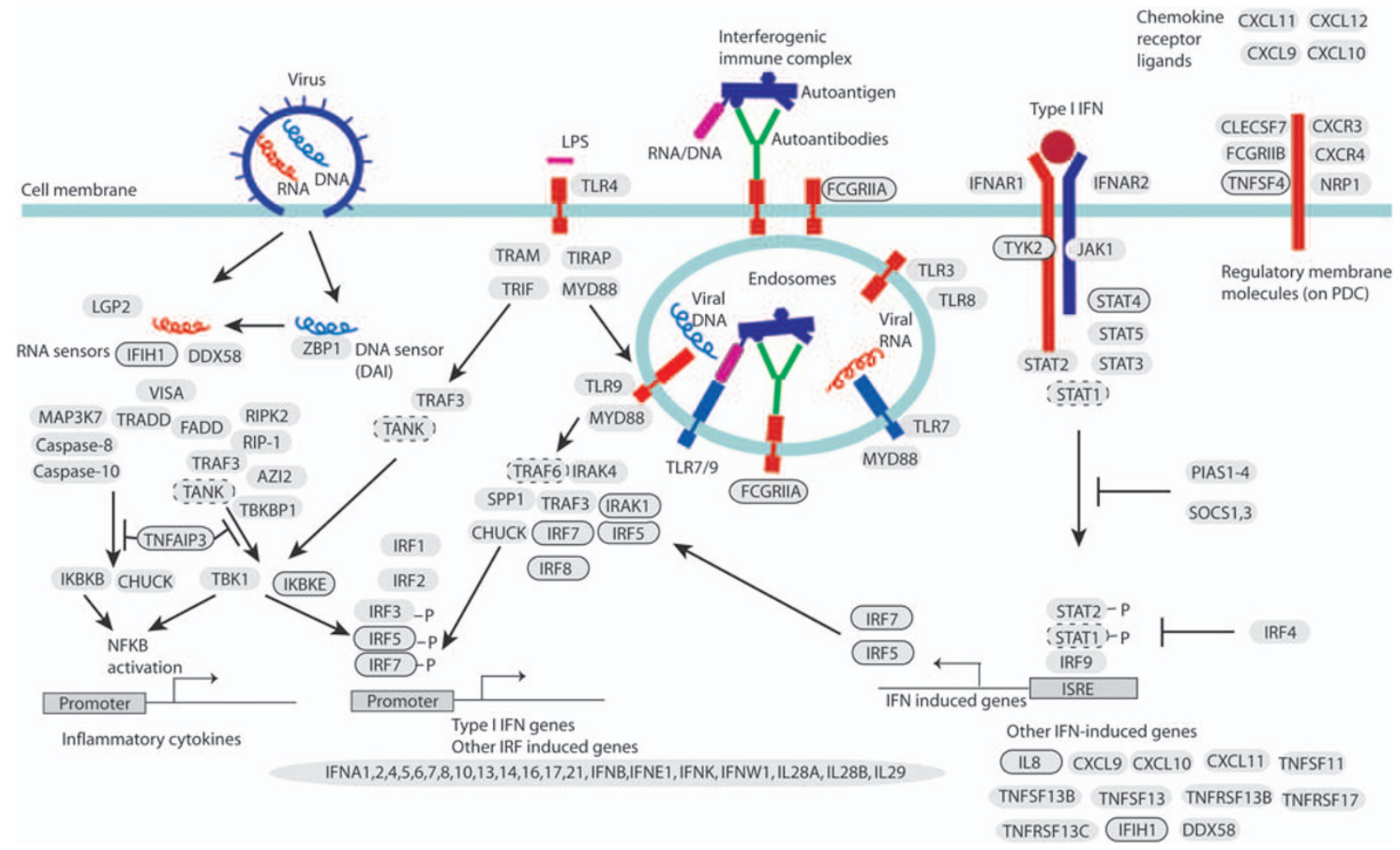

Figure 2 A schematic illustration of signalling within the type I interferon (IFN) system. Circled gene names have confirmed association to SLE: STAT4, IRF5, IRF7, IRF8, TNFAIP3 (A20), TNFSF4 (OX4OL), FCGR2A, TYK2, IFIH1 (MDA5), IRAK1, as well as IKBKE and IL8, identified in this study. Genes with dashed circles are TANK and TRAF6, which we find associated with SLE in the Swedish but not US cohorts, and STAT1 that has an association signal, which cannot be distinguished from that of STAT4. Left: Induction of inflammatory cytokines and type I IFN genes in response to viral RNA/DNA by the helicases IFIH1 (MDA5), DDX58 (RIG-I) and the DNA sensor ZBP1 (DAI), or by bacterial LPS through toll-like receptor 4. These pathways signal using TANK that interacts with IKBKE or TBK1, which together can mediate phosphorylation of the interferon regulatory factors IRF3 and 7, leading to the transcription of type I IFN genes. Activation of this pathway can be inhibited by TNFAIP3 (A20). Middle: Induction of IFN production in plasmacytoid dendritic cells (pDCs) by endogenous immune complexes. Immune complexes are endocytosed through FCGRIIA, which leads to activation of TLR7/9. After a signalling cascade, which includes IRAK1 and TRAF6, the interferon regulatory factors IRF5 and IRF7 are activated leading to the transcription of type I IFN genes. Also IRF8 can contribute to this activation. Right: IFN signalling through the type I IFN receptor IFNAR. Association of type I IFN to the receptor activates the kinases TYK2 and JAK1, which signal through STAT1 and STAT2. Also other STATs, including STAT4, are activated in this process. The STAT1/STAT2 complex associates with IRF9, which in turn binds to interferon-stimulated response elements (ISREs) and induces expression of IFN-induced genes, such as IRF5, IRF7 and indirectly IL8. Far right: Membrane proteins expressed on pDCs. TNFSF4 (OX40 L), together with endogenous type I IFNs provide a means for the pDCs to control the T-cell response, specifically to profile it towards a $T_{H} 1$ response. Activation of the NF $k$ B pathway, and thus production of inflammatory cytokines, also occurs through TLR7/9 (not shown). Proteins are denoted by their corresponding gene names.

perfectly correlated with IL8 rs4694178 (rs4694636, $r^{2}=1$ HapMap $\mathrm{CEU}$ ) was tested, and showed an odds ratio suggestive of association with SLE (OR CI: 1.01-1.49, dbGaP). The modest power to detect an effect of this size, or genetic heterogeneity between populations could explain these conflicting results.

Although the TANK and TRAF6 genes, which gave clear association signals $(P<0.001)$ in the Swedish cohort, did not replicate in the US GWAS data, these genes remain interesting candidates. TANK signals immediately upstream of IKK $\varepsilon$ in the TLR4- and IFIH1/ DDX58-mediated activation of type I IFNs and inflammatory cytokines in response to bacterial and viral stimuli, respectively (Figure 2). Association of TANK with SLE would thus further support an important role for the RLR pathway in SLE. TRAF6 is a ubiquitin ligase that mediates signal transduction from, for example, members of the TLR family leading to activation of NF $\kappa$ B and IRFs. Polyubiquitination of IRF5 after its interaction with IRAK1 is mediated by TRAF6, which enables IRF5 to translocate to the nucleus and exert its effect on gene expression. On the other hand, TNFAIP3 can inhibit TLR-induced activity of $\mathrm{NF} \kappa \mathrm{B}$ by de-ubiquitination of
TRAF6. ${ }^{35}$ Interestingly, also IRAK1 has been associated with risk for SLE, ${ }^{36}$ and because SNPs in the TRAF6 region and in TNFAIP3 have also been associated with risk for RA, ${ }^{37-39}$ it seems as variants of all these genes have the potential to contribute to loss of tolerance and autoimmune reactions. Further studies will be needed to determine whether the TANK and TRAF6 genes have an effect on SLE, and whether this effect is specific for Scandinavian populations.

Our study confirms the important role of the type I IFN system in SLE, and suggests multiple genes from this pathway as candidates for functional studies and as interesting therapeutic targets (Figure 2). These results also point more specifically to the importance of genes in the RLR pathway, which is activated in response to viral infections because of the ability of IFIH1/DDX58 to recognise cytoplasmic viral RNA. This pathway is active in cells other than the pDCs, including monocyte derived dendritic cells. In addition to IFIH1, factors such as TANK, IKBKE and TNFAIP3 contribute to signalling in the RLR pathway (reviewed in ${ }^{40}$ ). However, there is also evidence for involvement in SLE of the MYD88-dependent pathway activated by endosomal TLR7/9 by RNA/DNA from dying cells, immune complexes 
(IC) or by viral RNA/DNA, because IRF8, IRAK1, FCGR2A and potentially TRAF6, in this pathway are associated with SLE. Thus, at least two pathways seem important in SLE, both leading to production of type I IFN and inflammatory cytokines through activation of IRF3, IRF5 and IRF7, and additional transcriptions factors, especially NF $\kappa \mathrm{B}$. Association to SLE has further been demonstrated for the type I IFN signalling through the IFNAR, specifically for TYK2 and STAT4, as well as the IFN-regulated genes IFIH1, IRF5, IRF7 and IL8. Consequently, a large number of genes, located in different functional parts of the type I IFN system, are associated with SLE indicating a general role for the type I IFN system genes in autoimmunity.

\section{CONFLICT OF INTEREST}

Robert R Graham and Timothy W Behrens are employees of Genentech Corp. The other authors declare no conflict of interest.

\section{ACKNOWLEDGEMENTS}

This study was supported by a Target Identification in Lupus (TIL) grant from the Alliance for Lupus Research, US, the Swedish Research Council for Medicine, the Knut and Alice Wallenberg Foundation, the Swedish Rheumatism Foundation, the King Gustaf V 80-year Foundation, COMBINE, the European Community 6th Framework Program (LSHM-CT-2007-037273), the Swedish Heart-Lung Foundation, the Torsten and Ragnar Söderberg Foundation, the 'Visare Norr' Fund for Northern County Councils of Sweden, The Åke Wiberg Foundation, ALF funding from Stockholm County Council and Karolinska Institutet, the National Institutes of Health (R01 AR44804, K24 AR02175), the National Center for Research Resources (5 M01 RR00079), and a Kirkland Scholar Award to LAC. We thank Rezvan Kiani Dehkordi at the Rheumatology Clinic, Uppsala University Hospital, for assistance with DNA sample collection. Professor Göran Hallmans, Head of the Medical Biobank of Northern Sweden for providing blood samples. Ann-Christin Wiman, Caisa Pöntinen, Molecular Medicine, Department of Medical Sciences, Uppsala University, for assistance with genotyping. Genotyping was performed using equipment available at the SNP Technology Platform in Uppsala, Sweden (http://www.genotyping.se).

1 Sigurdsson S, Nordmark G, Goring $\mathrm{HH}$ et al: Polymorphisms in the tyrosine kinase 2 and interferon regulatory factor 5 genes are associated with systemic lupus erythematosus. Am J Hum Genet 2005; 76: 528-537.

2 Remmers EF, Plenge RM, Lee AT et al: STAT4 and the risk of rheumatoid arthritis and systemic lupus erythematosus. N Engl J Med 2007; 357: 977-986.

3 Graham RR, Hom G, Ortmann W, Behrens TW: Review of recent genome-wide association scans in lupus. J Intern Med 2009; 265: 680-688.

4 Gateva V, Sandling JK, Hom G et al: A large-scale replication study identifies TNIP1, PRDM1, JAZF1, UHRF1BP1 and IL10 as risk loci for systemic lupus erythematosus. Nat Genet 2009; 41: 1228-1233.

5 Hooks JJ, Moutsopoulos HM, Geis SA, Stahl NI, Decker JL, Notkins AL: Immune interferon in the circulation of patients with autoimmune disease. N Engl J Med 1979; 301: 5-8.

6 Bengtsson AA, Sturfelt G, Truedsson L et al: Activation of type I interferon system in systemic lupus erythematosus correlates with disease activity but not with antiretroviral antibodies. Lupus 2000; 9: 664-671.

7 Baechler EC, Batliwalla FM, Karypis G et al: Interferon-inducible gene expression signature in peripheral blood cells of patients with severe lupus. Proc Natl Acad Sci USA 2003; 100: 2610-2615.

8 Ronnblom LE, Alm GV, Oberg KE: Possible induction of systemic lupus erythematosus by interferon-alpha treatment in a patient with a malignant carcinoid tumour. J Intern Med 1990; 227: 207-210.

9 Wallace DJ, Petri M, Olsen N et al: MEDI-545, an anti-interferon alpha monoclonal antibody, shows evidence of clinical activity in systemic lupus erythematosus. Arthritis Rheum 2007; 56(Suppl 9): S526-S527.

10 Yao Y, Richman L, Higgs BW et al: Neutralization of interferon-alpha/beta-inducible genes and downstream effect in a phase I trial of an anti-interferon-alpha monoclonal antibody in systemic lupus erythematosus. Arthritis Rheum 2009; 60: 1785-1796.

11 Tan EM, Cohen AS, Fries JF et al: The 1982 revised criteria for the classification of systemic lupus erythematosus. Arthritis Rheum 1982; 25: 1271-1277.

12 Bell PA, Chaturvedi S, Gelfand CA et al: SNPstream UHT: ultra-high throughput SNP genotyping for pharmacogenomics and drug discovery. Biotechniques 2002; (Suppl 74), 70-72, 76-77.

13 Broadbent HM, Peden JF, Lorkowski S et al: Susceptibility to coronary artery disease and diabetes is encoded by distinct, tightly linked SNPs in the ANRIL locus on chromosome 9p. Hum Mol Genet 2008; 17: 806-814.

14 Hom G, Graham RR, Modrek B et al: Association of systemic lupus erythematosus with C8orf13-BLK and ITGAM-ITGAX. N Engl J Med 2008; 358: 900-909.

15 Marchini J, Howie B, Myers S, McVean G, Donnelly P: A new multipoint method for genome-wide association studies by imputation of genotypes. Nat Genet 2007; 39: 906-913.

16 Price AL, Patterson NJ, Plenge RM, Weinblatt ME, Shadick NA, Reich D: Principal components analysis corrects for stratification in genome-wide association studies. Nat Genet 2006; 38: 904-909.

17 Purcell S, Neale B, Todd-Brown $\mathrm{K}$ et al: PLINK: a tool set for whole-genome association and population-based linkage analyses. Am J Hum Genet 2007; 81: 559-575.

18 Sigurdsson S, Nordmark G, Garnier S et al: A risk haplotype of STAT4 for systemic lupus erythematosus is over-expressed, correlates with anti-dsDNA and shows additive effects with two risk alleles of IRF5. Hum Mol Genet 2008; 17: 2868-2876.

19 Sharma S, tenOever BR, Grandvaux N, Zhou GP, Lin R, Hiscott J: Triggering the interferon antiviral response through an IKK-related pathway. Science 2003; 300: 1148-1151.

20 Sankar S, Chan H, Romanow WJ, Li J, Bates RJ: IKK-i signals through IRF3 and NFkappaB to mediate the production of inflammatory cytokines. Cell Signal 2006; 18: 982-993.

21 Graham RR, Cotsapas C, Davies L et al: Genetic variants near TNFAIP3 on 6q23 are associated with systemic lupus erythematosus. Nat Genet 2008; 40: 1059-1061.

22 Musone SL, Taylor KE, Lu TT et al: Multiple polymorphisms in the TNFAIP3 region are independently associated with systemic lupus erythematosus. Nat Genet 2008; 40: 1062-1064

23 tenOever BR, Ng SL, Chua MA, McWhirter SM, Garcia-Sastre A, Maniatis T: Multiple functions of the IKK-related kinase IKKepsilon in interferon-mediated antiviral immunity. Science 2007; 315: 1274-1278.

24 Dieguez-Gonzalez R, Akar S, Calaza M et al: Genetic variation in the nuclear factor kappaB pathway in relation to susceptibility to rheumatoid arthritis. Ann Rheum Dis 2009; 68: 579-583.

25 Corr M, Boyle DL, Ronacher L, Flores N, Firestein GS: Synergistic benefit in inflammatory arthritis by targeting I kappaB kinase epsilon and interferon beta. Ann Rheum Dis 2009; 68: 257-263.

26 Harley JB, Alarcon-Riquelme ME, Criswell LA et al: Genome-wide association scan in women with systemic lupus erythematosus identifies susceptibility variants in ITGAM, PXK, KIAA1542 and other loci. Nat Genet 2008; 40: 204-210.

27 Santer DM, Yoshio T, Minota S, Moller T, Elkon KB: Potent induction of IFN-alpha and chemokines by autoantibodies in the cerebrospinal fluid of patients with neuropsychiatric lupus. J Immunol 2009; 182: 1192-1201.

28 Manuse MJ, Parks GD: TLR3-dependent upregulation of RIG-I leads to enhanced cytokine production from cells infected with the parainfluenza virus SV5. Virology 2010; 397: 231-241.

29 Holcombe RF, Baethge BA, Wolf RE et al: Correlation of serum interleukin-8 and cell surface lysosome-associated membrane protein expression with clinical disease activity in systemic lupus erythematosus. Lupus 1994; 3: 97-102.

30 Trysberg E, Carlsten H, Tarkowski A: Intrathecal cytokines in systemic lupus erythematosus with central nervous system involvement. Lupus 2000; 9: 498-503.

31 Lit LC, Wong CK, Tam LS, Li EK, Lam CW: Raised plasma concentration and ex vivo production of inflammatory chemokines in patients with systemic lupus erythematosus. Ann Rheum Dis 2006; 65: 209-215.

32 Rovin BH, Lu L, Zhang X: A novel interleukin-8 polymorphism is associated with severe systemic lupus erythematosus nephritis. Kidney Int 2002; 62: 261-265.

33 Huang CM, Huo AP, Tsai CH, Chen CL, Tsai FJ: Lack of association of interleukin- 6 and interleukin-8 gene polymorphisms in Chinese patients with systemic lupus erythematosus. J Clin Lab Anal 2006; 20: 255-259.

34 Sanchez E, Sabio JM, Callejas JL et al: Association study of genetic variants of pro-inflammatory chemokine and cytokine genes in systemic lupus erythematosus. BMC Med Genet 2006; 7: 48.

35 Boone DL, Turer EE, Lee EG et al: The ubiquitin-modifying enzyme A20 is required for termination of Toll-like receptor responses. Nat Immunol 2004; 5: 1052-1060.

36 Jacob CO, Zhu J, Armstrong DL et al: Identification of IRAK1 as a risk gene with critical role in the pathogenesis of systemic lupus erythematosus. Proc Natl Acad Sci USA 2009; 106: 6256-6261.

37 Raychaudhuri S, Thomson BP, Remmers EF et al: Genetic variants at CD28, PRDM1 and CD2/CD58 are associated with rheumatoid arthritis risk. Nat Genet 2009; 41: $1313-1318$

38 Thomson W, Barton A, Ke X et al: Rheumatoid arthritis association at 6q23. Nat Genet 2007; 39: 1431-1433.

39 Plenge RM, Cotsapas C, Davies $L$ et al: Two independent alleles at 6q23 associated with risk of rheumatoid arthritis. Nat Genet 2007; 39: 1477-1482.

40 Takeuchi O, Akira S: MDA5/RIG-I and virus recognition. Curr Opin Immunol 2008; 20: 17-22.

(c)

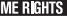

Attribution-NonCommercial-No Derivative Works 3.0 Unported Licence. To view a copy of this licence, visit http:// creativecommons.org/licenses/by-nc-nd/3.0/

Supplementary Information accompanies the paper on European Journal of Human Genetics website (http://www.nature.com/ejhg) 